\title{
Tisserandes fatales (Apulée) et Fées de Cour (Perrault) : Le sort difficile d'une Belle «née pour être couronnée »
}

\section{Ute Heidmann}

\author{
(2) OpenEdition \\ Journals \\ Édition électronique \\ URL : http://journals.openedition.org/edl/202 \\ DOI : 10.4000/edl.202 \\ ISSN : 2296-5084 \\ Éditeur \\ Université de Lausanne

\section{Édition imprimée} \\ Date de publication : 15 décembre 2011 \\ Pagination : 205-220 \\ ISBN : 978-2-940331-26-0 \\ ISSN : 0014-2026
}

Référence électronique

Ute Heidmann, «Tisserandes fatales (Apulée) et Fées de Cour (Perrault) : Le sort difficile d'une Belle «née pour être couronnée » », Études de lettres [En ligne], 3-4 | 2011, mis en ligne le 15 décembre 2014, consulté le 18 décembre 2020. URL : http://journals.openedition.org/edl/202 ; DOI : https://doi.org/ 10.4000/edl.202 


\section{TISSERANDES FATALES (APULÉE) ET FÉES DE COUR (PERRAULT) : LE SORT DIFFICILE D'UNE BELLE "NÉE POUR ÊTRE COURONNÉE"}

Cette étude s'attache à analyser la façon dont Charles Perrault conçoit La Belle au bois dormant en «reconfigurant» le conte ancien de Psyché inséré dans les Métamorphoses d'Apulée. Dans un «dialogue intertextuel» très subtil avec la célèbre fabella, Perrault crée un conte moderne qui tire ses effets de sens des différences très subtiles qu'il introduit par rapport à cet intertexte. C'est par le biais d'une analyse comparative proche de la lettre des textes dans leur forme et langue d'origine que ces différences apparaissent et prennent leur signification. Une telle analyse montre que le rôle que les fées prennent dans le destin de la Belle est plus ambigu qu'il n'y paraît à première vue. Le conte s'avère en fait sous-tendu par une vision très critique de la société aristocratique qui destine ses filles à des princes inconnus à la généalogie douteuse (susceptibles d'avoir des mères «de race Ogresse»). Ce sens caché et subversif «se découvre plus ou moins, selon le degré de pénétration de ceux qui le[s] lisent", comme le recommande l'épître dédicatoire à Mademoiselle, nièce de Louis XIV et "née pour être couronnée», elle-même victime potentielle de la politique maritale dénoncée.

Ma contribution au présent volume s'attache à analyser la façon dont Charles Perrault crée La Belle au bois dormant, premier conte des Histoires ou contes du temps passé. Avec des Moralitez de 1697, en "reconfigurant" le conte ancien de Psyché inséré dans les Métamorphoses d'Apulée ${ }^{1}$. Dans la

I. Une analyse comparative que j'ai proposé d'appeler "différentielle» permet de montrer que les contes de Perrault, loin de puiser dans une hypothétique tradition "populaire» (comme le présupposent notamment les travaux de Soriano, Delarue et Robert indiqués dans la bibliographie ci-jointe) relèvent d'un dialogue très complexe avec les genres et textes des littératures européennes, anciennes et modernes. Je présente cette méthode et les analyses comparatives qui étayent ces hypothèses dans les travaux indiqués dans la bibliographie ci-jointe. De telles analyses comparatives des 
préface programmatique de son recueil Griselidis, nouvelle avec le conte de Peau d'Asne, et celuy des Souhaits ridicules de 1694, Perrault compare les genres anciens et modernes. Selon lui, les contes anciens et les siens sont de la même "espèce» en ce qui concerne leur dispositif énonciatif et narratif:

La Fable de Psiché écrite par Lucien \& par Apulée, est une fiction toute pure $\&$ et un conte de Vieille come celuy de Peau D'Asne 2 .

Cette phrase est parfois citée pour démontrer que le défenseur des Modernes "connaît décidément mal ses classiques", parce que "l'on trouve la fable de Psyché chez Apulée [...] et non pas chez Lucien» ${ }^{3}$. Ce reproche est selon moi infondé: la remarque de Perrault témoigne au contraire de sa conscience aiguë de la complexité du dispositif narratif du roman d'Apulée et de celui de la fabella. Dans son Traité sur l'origine des romans, Jean-Daniel Huet avait formulé une hypothèse concernant le rôle du court texte grec Loukios ê Onos (Lucius ou l'âne) attribué à Lucien de Samostate dans le roman d'Apulée. Selon Huet, Lucien est l'inventeur du dispositif du narrateur-âne Lucius par lequel il aurait représenté un auteur du nom de Lucius de Patras pour ridiculiser sa crédulité .

Lorsque Perrault relève la double écriture de la Fable de Psyché par "Lucien \& Apulée» et donc la complexité du dispositif narratif, il se fonde à mon avis sur cette explication de la genèse du roman d'Apulée par Huet. Même si Lucien n'a pas inventé l'histoire de Psyché, il est considéré comme l'inventeur du dispositif énonciatif multiple qui fait raconter à un autre (à Lucius métamorphosé en âne) ce qu'il dit avoir entendu, comme le souligne Perrault, «raconter par une vieille femme, à une fille que des voleurs avoient enlevée». Son explicitation du dispositif énonciatif

contes de Perrault avec la fabella de Psyché d'Apulée, qui n'ont pas encore été menées de façon systématique, infirment les hypothèses du folkloriste Jan-Öjwind Swahn, The Tale of Cupid and Psyche (1955), qui considère le conte de Psyché uniquement en termes de "tale-type», ouvrage déjà critiqué par Fehling en 1977. L'ouvrage de Graham, Fairytale in the Ancient World (2000) reste également déterminé par les présupposés des folkloristes sans procéder à aucune étude intertextuelle précise. Les commentateurs des contes de Perrault (dont Rouger, Collinet et Escola) notent occasionnellement des ressemblances au niveau de certains "motifs", sans toutefois procéder à des études comparatives systématiques des textes latins et français.

2. Ch. Perrault, Griselidis, nouvelle avec le conte de Peau d'Asne, "Préface», sans pagination.

3. Cf. Ch. Perrault, Contes de Charles Perrault, éd. M. Escola, p. 41.

4. P.-D. Huet, Lettre-traité de Pierre-Daniel Huet sur l'origine des romans, p. 74-75. 
des Métamorphoses est importante, car elle signale que ni le conte ancien d'Apulée ni encore ses propres contes ne sont des "contes de Vieille", comme on persiste à le croire. Le commentaire de Perrault insiste sur le fait que l'auteur ancien et lui-même, auteur moderne, se servent d'un même dispositif complexe qui les fait ressembler à ce type de récit.

Perrault souligne cette ressemblance entre le conte ancien d'Apulée et le sien dans la préface de 1694, mais il insiste aussi sur une différence importante qui se situe sur un autre plan de comparaison: la fonction attribuée au conte dans l'Antiquité et à l'époque moderne. Il ne manque pas d'affirmer que ses contes ne sont pas de "pures» bagatelles, que le «récit enjoüé» qui «enveloppe» ces bagatelles n'est choisi «que pour les faire entrer plus agréablement dans l'esprit \& d'une maniere qui instruisist $\&$ divertist tout ensemble ${ }^{5}$. Cette dimension instructive distingue selon lui ses propres contes de la «Fable de Psiché» et des «Fables Milésiennes» destinées à divertir sans pour autant instruire. Contrairement à ce que la pratique courante des interprétations allégorisantes de la fable de Psyché pouvait laisser entendre ${ }^{6}$, la «Fable de Psiché» ne contient pas, précise-t-il, de «Morale cachée» destinée à instruire les lecteurs:

A l'égard de la Morale cachée dans la Fable de Psiché, Fable en ellemesme très agréable $\&$ tres ingénieuse, je la compareray avec celle de Peau-d'Asne quand je la sçaurai, mais jusques icy je n'ay pû la deviner. Je sçay bien que Psiché signifie l'Ame; mais je ne comprens point ce qu'il faut entendre par l'Amour qui est amoureux de Psiché, c'est-àdire de l'Ame, encore moins ce qu'on ajoûte, que Psiché devoit estre heureuse, tant qu'elle ne connoistraist point celuy dont elle estoit aimée, qui estoit l'Amour, mais qu'elle seroit tres malheureuse dès le moment qu'elle viendroit à le connoistre: voilà pour moy une enigme impenetrable ${ }^{7}$.

5. Ch. Perrault, Griselidis, nouvelle avec le conte de Peau d'Asne, "Préface», sans pagination.

6. Au sujet de la tradition de l'interprétation allégorisante du texte d'Apulée, voir la remarquable étude de Véronique Gély, L'invention d'un mythe. L'ouvrage fait état de l'ironie de la préface de Perrault à l'égard de cette tradition allégorisante. L'analyse des relations intertextuelles précises que les contes de Perrault entretiennent avec le conte de Psyché n'a en revanche pas encore été menée.

7. Ch. Perrault, Griselidis, nouvelle avec le conte de Peau d'Asne, "Préface», sans pagination. 
Perrault dénonce ici l'incompréhensible logique d'une interprétation allégorisante. A partir de ce diagnostic, il s'engage selon moi dans un projet poétique particulièrement audacieux qui consiste à recourir à la «Fable en elle-mesme très agréable $\&$ tres ingénieuse» d'Apulée, pour en faire des contes modernes dotés, eux, d'une «morale cachée», c'est-à-dire d'une dimension instructive et utile. Le défenseur des Modernes relève ainsi un défi qu'il avait formulé de façon ironique dans la préface de 1694 :

Mais comme j'ay affaire à bien des gens qui ne se payent pas de raisons $\&$ qui ne peuvent estre touchez que par l'autorité \& par l'exemple des Anciens, je vais les satisfaire là-dessus ${ }^{8}$.

Au lieu d' "imiter» les Anciens selon les prescriptions du chef de file des Anciens, Nicolas Boileau, que Perrault venait de disqualifier comme relevant d'une "imitation mal endenduë des Anciens " ${ }^{9}$, il oppose à son adversaire une autre façon de recourir aux Anciens. Au lieu de suivre l'intrigue de la fabella antique, comme la doxa l'exigeait, il invente un tout autre procédé. Il puise dans le texte latin des éléments narratifs, stylistiques et génériques pour «fabriquer" de nouvelles histoires dont celles du Petit chaperon rouge et de La Barbe bleue ${ }^{10}$, mais aussi celle de La Belle au bois dormant.

La Belle au bois dormant n'hérite pas seulement du surnom (pulchra) de la Psyché d'Apulée. Tout en dialoguant avec la célèbre fabella et d'autres textes latins, italiens et français, Perrault crée un conte moderne qui tire ses effets de sens des différences très subtiles qu'il introduit par rapport à ces intertextes. C'est par le biais d'une analyse comparative des textes impliqués, à partir de leurs langues d'origine, que ces différences apparaissent et prennent leur signification. Comme il est impossible, dans le cadre restreint de cet article, de déployer toute la complexité de ce véritable palimpseste ${ }^{11}$ et de la façon dont Perrault y inscrit une

8. Ibid., «Préface», sans pagination.

9. Ch. Perrault, L'Apologie des femmes, p. 3-4.

Io. J'ai analysé ce procédé pour Le Petit Chaperon rouge et de La Barbe bleue dans Textualité et intertextualité des contes, p. 81-152.

II. Pour une analyse plus extensive qui inclut aussi les autres intertextes notamment ceux de Basile (qui recourt à son tour au roman de Perceforest) et ceux de La Fontaine, je me permets de renvoyer au chapitre «La Belle au bois dormant palimpseste" dans mon ouvrage Pour une comparaison différentielle. Mythes et contes, à paraitre aux Editions Classiques Garnier. 
autre histoire, je me limite ici à l'analyse de la confrontation de la Belle avec la fileuse fatale et du rôle que les fées y prennent, comme le sujet du présent volume le requiert. Cette scène s'inscrit, selon moi, sur le fond intertextuel de la descente aux enfers que Vénus inflige à Psyché pour se débarrasser de la trop belle mortelle dont son fils Cupidon est tombé amoureux. Lorsque Psyché cherche à se jeter du haut d'une tour par désespoir, celle-ci s'anime pour lui donner ces conseils bienveillants destinés à guider sa traversée des enfers:

Transito fluuio modicum te progressam textrices orabunt anus telam struentes manus paulisper accommodes, nec id tamen tibi contingere fas est. Nam haec omnia tibi et multa alia de Veneris insidiis orientur, ut uel unam de manibus omittas offulam. Nec putes futile istud polentacium damnum leue; altera enim perdita lux haec tibi prorsus denegabitur.

Passé le fleuve, quand tu auras un peu marché, de vieilles tisserandes en train de monter leur fil de chaîne te demanderont un petit coup de main, mais ça non plus, ne pas toucher, danger parce que tout ça et bien d'autres encore, c'est des pièges tendus par Vénus, pour que tu laisses au moins une galette t'échapper des mains, et ne pense pas que ce soit une broutille et une mince perte cette polentasse, parce qu'une galette de perdue et c'est la lumière du jour de confisquée pour toi ${ }^{12}$.

La jeune femme reprend courage et exécute scrupuleusement tout ce que lui a dit la tour. Le narrateur latin n'hésite pas d'énumérer à nouveau tout ce que Psyché devait faire et surtout ne pas faire:

Nec moratur Psyche pergit Taenarum sumptisque rite stipibus illis et offulis infernum decurrit meatum transitoque per silentium asinario debili et amnica stipe uetori data neglecto supernatantis mortui desiderio et spretis textricum subdolis precibus et offulae cibo sopita canis horrenda rabie domum Proserpinae penetrat.

Sans tarder Psyché alla à Ténare, se munit des oboles rituelles et des galettes, descendit le couloir des Enfers, passa en silence devant l'ânier infirme, donna au nocher l'obole du péage fluvial, négligea la demande du mort nageur, méprisa les sournoises suppliques des tissandières, endormit d'une galette en pâture l'horrible rage du chien, $[\ldots]^{13}$.

I2. Apulée, Les Métamorphoses ou l'Ane d'or, p. 234-235.

I3. Ibid., p. 236-237. 
Voici ce que fait la Belle de Perrault dans la scène décisive qui lui fait rencontrer la fileuse fatale:

Au bout de quinze ou seize ans, le Roi \& la reine estant allez à une de leurs Maisons de plaisance, il arriva que la jeune Princesse courant un jour dans le Château, \& montant de chambre en chambre, alla jusqu'en haut d'un donjon dans un petit galetas, où une bonne Vieille estoit seule à filer sa quenoüille ${ }^{14}$.

Si nous examinons cette scène sur la toile de fond de la rencontre avec les tisserandes infernales, il est flagrant que la Belle du conte de Perrault fait exactement ce que Psyché avait réussi à ne pas faire:

Que faites-vous là, ma bonne femme, dit la Princesse: je file, ma belle enfant, luy répondit la vieille qui ne la connoissait pas. Ha! que cela est joli, reprit la Princesse, comment faites-vous? donnez-moy que je voye si j'en ferois bien autant ${ }^{15}$.

La princesse ne parle pas seulement sans précaution, mais elle touche aussi aux outils de la fileuse, ce que sa sœur intertextuelle avait eu la plus stricte interdiction de faire: "Ne pas toucher, danger", avait dit la tour à Psyché. La Belle de Perrault y touche pour une raison assez simple dont les nombreux interprètes du conte font étonnamment peu état: elle ne sait pas qu'il est dangereux de toucher à un fuseau ${ }^{16}$. Si elle ne le sait pas, c'est que personne ne le lui a dit. Le donjon dans lequel elle monte comme Psyché sur la tour, ne s'anime pas pour lui parler: il reste silencieux et un simple élément du décor. Plus étrange encore, la Belle n'a reçu aucun conseil de la part du Roi son père ou de la Reine sa mère, ce qui paraît d'autant plus étonnant qu'ils sont responsables de la malédiction qui pèse sur leur fille. Leur oubli de la vieille Fée au moment du baptême a été la cause de sa vengeance qui a pris pour cible l'innocente petite fille. Le narrateur avait certes donné comme raison "qu'il y avoit plus de cinquante ans qu'elle n'estoit sortie d'une Tour,

14. Ch. Perrault, Histoires ou contes du temps passé, p. 9-10.

I5. Ibid., p. 10-11.

I6. De façon analogue, le Petit Chaperon rouge dans le conte qui suit La Belle au bois dormant parle au Loup, parce que ni sa mère et sa Mère-grand lui avaient dit qu'il était dangereux de parler avec un loup. Une analyse plus approfondie de cet aspect se trouve dans U. Heidmann, J.-M. Adam, Textualité et intertextualité des contes, p. 81-87. 
\& qu'on la croyait morte, ou enchantée ${ }^{17}$. Mais le fait de se fier à une simple rumeur sans procéder à aucune vérification témoigne d'une attitude pour le moins insouciante de la part d'un souverain. Le narrateur souligne à plusieurs reprises cette étonnante capacité du roi d'oublier ou de se souvenir trop tard. Le jour de l'événement fatal, le roi et la reine, qui étaient allés à leur maison de plaisance, en sont rappelés au moment de l'incident: "Alors le Roy, qui estoit monté au bruit, se souvint de la prédication des Fées $[\ldots]{ }^{18}$. Ni l'un ni l'autre, qui avaient pourtant tellement souhaité sa naissance, n'avaient trouvé utile de renseigner la principale intéressée sur ce qui risquerait de lui arriver après l'incident diplomatique survenu à son baptême. Son père avait pourtant pris de grandes mesures "politiques» juste après le baptême:

Le Roi pour tâcher d'éviter le malheur annoncé par la vieille, fit publier aussi tost un Edit, par lequel il deffendoit à toutes personnes de filer au fuseau, ny d'avoir des fuseaux chez soy sur peine de la vie ${ }^{19}$.

Cet édit royal interdisant à tous les sujets d'exercer une activité économique élémentaire s'avère en fait totalement inefficace. Il aurait été plus utile de prévenir la princesse du péril qui la guettait, comme l'avait fait la tour dans la fabella latine. La mesure prise par le Roi (qui devait rappeler aux lecteurs contemporains les nombreux édits que Louis XIV publiait à toute occasion) s'avère en effet doublement inutile, parce qu'elle n'a pas été exécutée de façon conséquente: une fois encore une vieille a été oubliée dans une tour. Comme le narrateur ne manque pas de le souligner, ce n'est pas seulement la jeune fille qui ignore tout du danger qui la guette, mais c'est aussi la fileuse: "Cette bonne femme n'avoit point ouï parler des deffenses que le Roi avoit faites de filer au fuseau» ${ }^{20}$. Le roi et la reine vaquent en revanche à leurs plaisirs: le jour de la rencontre fatale de la princesse ignorante avec la fileuse tout aussi ignorante, le Roi et la Reine étaient allés "à une de leurs Maisons de plaisance» ${ }^{21}$, ne veillant ainsi pas du tout sur leur fille. A la différence notable de sa sœur

\footnotetext{
17. Ch. Perrault, Histoires ou contes du temps passé, p. 4.

I8. Ibid., p. 12.

19. Ibid., p. 9.

2o. Ibid., p. 10.

2I. Ibid., p. 9.
} 
intertextuelle, la protagoniste du conte moderne est donc seule, sans conseil aucun et parfaitement ignorante de ce qui peut lui arriver.

On pourrait objecter à cela que la Belle est au contraire bien mieux protégée que Psyché, parce qu'au lieu d'une seule, elle jouit de la bienveillance de sept fées, dont elle a reçu, le jour de son baptême, "toutes les perfections imaginables» ${ }^{22}$ :

La plus jeune luy donna pour don qu'elle seroit la plus belle personne du monde, celle d'après qu'elle auroit de l'esprit comme un Ange, la troisième qu'elle auroit une grace admirable à tout ce qu'elle feroit, la quatrième qu'elle danseroit parfaitement bien, la cinquième qu'elle chanteroit comme un Rossignol, \& la sixiéme qu'elle joüeroit de toutes sortes d'instrumens dans la dernière perfection ${ }^{23}$.

Si nous lisons cette liste des perfections octroyées à la fille du roi à la lumière de ce qu'elle devra affronter dans sa vie de jeune femme mariée à un prince inconnu dont elle ignore qu'il est fils d'une ogresse, nous devons nous rendre à l'évidence que "toutes ces perfections imaginables» dont elle a été si solennellement dotée, ne lui servent à rien. Face à une belle-mère à laquelle son mari princier l'expose sans avertissement, ni ses talents de musicienne et de danseuse, ni encore le fait qu'elle soit la plus belle personne du monde et encore moins "son esprit d'Ange» ne pourront la protéger. Face au pouvoir destructeur de la reine mère, l'ignorante princesse de l'Ancien Régime est plus démunie que Psyché qui a été mise au courant des moyens à mettre en œuvre pour déjouer les pièges de sa belle-mère.

Les élégantes fées françaises s'avèrent très conformistes dans le choix de leurs dons. Les qualités et les talents dont elles dotent la princesse sont exactement ceux que l'étiquette de la Cour demandait aux jeunes femmes de qualité en âge de se marier. Danser "parfaitement bien", "chanter comme un Rossignol", jouer «de toutes sortes d'instrumens dans la dernière perfection", et tout cela avec «un esprit d'ange» et avec une grâce admirable, ce sont en effet les talents requis des filles nobles

22. Ibid., p. 3. Notons que Perrault met une majuscule au mot Fée dès le recueil imprimé de 1697, majuscule que je reprends quand il est question de ce texte. Voir à ce sujet l'étude de Jean-Michel Adam "Quand les majuscules font sens", dans U. Heidmann, J.-M. Adam, Textualité et intertextualité des contes, p. 174-180.

23. Ch. Perrault, Histoires ou contes du temps passé, p. 7-8. 
sensées savoir entretenir agréablement leurs maris et la société noble sans se mêler de leurs affaires. Qu'en est-il de l'ultime don de la septième fée? Est-il apte à protéger la jeune fille contre le mauvais sort que l'oubli du roi son père lui a valu? L'analyse attentive de la façon dont elle formule son vou nous en dit plus:

Rassurez-vous, Roi \& Reine, vostre fille n'en mourra pas: il est vrai que je n'ay pas assez de puissance pour défaire entierement ce que mon ancienne a fait. La Princesse se percera la main d'un fuseau, mais au lieu d'en mourir, elle tombera seulement dans un profond sommeil qui durera cent ans, au bout desquels le fils d'un Roi viendra la réveiller ${ }^{24}$.

La Fée s'adresse de façon solennelle au couple royal qu'il lui importe avant tout de «rassurer». Elle déclare ne pas pouvoir annuler le verdict de celle qu'elle appelle "mon ancienne" et dont elle reconnaît ainsi les prérogatives de doyenne. Elle ne tentera pas non plus d'enseigner à la concernée ou à ses parents comment contourner le verdict par la ruse. Elle parvient en revanche à repousser le problème en annonçant que la princesse, lorsqu'elle sera en âge de se marier, tombera dans un sommeil qui durera cent ans. Si ce verdict "adouci» représente une consolation assez relative pour les parents affligés de se voir de nouveau privés de l'héritière de leur lignée qu'ils avaient si ardemment attendue, la Fée s'empresse de les rassurer sur ce point important. L'héritière unique du royaume, promet la "bonne Fée», sera réveillée par "le fils d'un Roi». Voilà de quoi rassurer les parents royaux. Leur fille, qui doit garantir la continuation de la lignée royale, sera réveillée, comprenons épousée par le fils d'un Roi. Elle deviendra donc reine, et quoi de plus important?

Perrault avait pris soin de rappeler cette règle chère aux familles de sang royal au tout début de son recueil que l'épître dédie à Mademoiselle, nièce de Louis XIV, fille de son frère, le duc d'Orléans et de la princesse Palatine. La toute première vignette représente un médaillon qui surplombe l'épître dédicatoire à Mademoiselle portant cette inscription: Pulcra et nata coronae, traduite sur le socle par:

Je suis belle et je suis née Pour estre couronnée

24. Ibid., p. 8. 
L'explicitation de cette devise à un endroit si stratégique du recueil prend une signification toute particulière, si on la relit sur le fond du premier conte, La Belle au bois dormant. Le lien entre la Belle du conte et Mademoiselle «belle» et «née pour être couronnée» avait été établi dans les vers finaux de la dédicace:

Et jamais Fée au temps jadis

Fit-elle à jeune Créature,

Plus de dons, \& de dons exquis,

Que vous en fait la Nature ${ }^{25}$.

L'inscription sur la vignette prend un sens plus aigu, si l'on sait ce que savent sans doute les lecteurs et lectrices familiers de la vie de la Cour. Mademoiselle est censée se marier enfin et son oncle, Louis XIV s'efforce de lui trouver un parti qui sert le mieux ses propres intérêts.

Après le don de la septième fée qui garantit aux parents le maintien de leur lignée, le roi n'a plus qu'à se préoccuper de la belle apparence et de la splendeur qui attestent son pouvoir à l'instar de son interdiction des fuseaux:

Alors le Roy, qui estoit monté au bruit, [...] fit mettre la Princesse dans le plus bel appartement du Palais, sur un lit de broderie d'or $\&$ d'argent ${ }^{26}$.

Lorsque la Fée arrive "dans un chariot tout de feu, traisné par les dragons ${ }^{27}$, le roi n'oublie pas de rendre hommage à celle qui sert si bien ses intérêts dynastiques. Très soucieux de l'étiquette (tout comme Louis XIV), "le Roy luy alla presenter la main à la descente du chariot " ${ }^{28}$. La Fée de son côté, nous dit le narrateur, «aprouva tout ce qu'il avoit fait; mais comme elle estoit grandement prévoyante, elle pensa que quand la Princesse viendroit à se réveiller, elle seroit bien embarassée toute seule dans ce vieux Château: voicy ce qu'elle fit " ${ }^{29}$. La Fée connaît et anticipe si bien les mœurs et coutumes de la Cour et de la famille royale qu'elle n'imagine pas d'autres détresses pour le réveil et l'avenir

\footnotetext{
25. Ibid., sans pagination.

26. Ibid., p. 12.

27. Ibid., p. 14.

28. Ibid., p. 14.

29. Ibid., p. 14.
} 
de la princesse que celle de se retrouver seule dans un vieux château. Elle emploie sa magie pour lui fournir le personnel qui, dès son réveil, lui garantira son confort habituel de princesse. La description excessivement détaillée des différentes fonctions de ce personnel se teinte d'ironie, si on la lit sur le fond des conseils tout aussi détaillés, mais autrement plus constructifs de la tour d'Apulée cités plus haut. Des Marmitons aux Gardes suisses en passant par les gouvernantes et le chien de compagnie appelé Pouffe (comme le petit chien de Mademoiselle), tout est prévu pour le confort de la princesse et future reine.

Il manque pourtant l'essentiel à ce scénario préparé par la bonne Fée en apparence si prévoyante: le mode d'emploi pour affronter les dangers qui guettent la Belle lors de son mariage avec le fils du roi promis par la Fée. La Princesse ignore en effet que ce fils de roi est aussi le fils d'une ogresse. Elle l'ignore, parce que personne ne le lui a dit et personne ne le lui dira par la suite, pas même son époux. Là encore, la princesse de l'Ancien Régime se trouve dans une situation bien plus difficile que Psyché si bien mise en garde par la tour qui n’avait laissé aucun doute sur les intentions meurtrières de Vénus à son égard.

La Fée de la Cour du Roi avait fait "un autre tour de son metier» consistant à faire croître "dans un quart-d'heure tout au tour du parc une si grande quantité de grands arbres $\&$ de petits, de ronces $\&$ d'épines entrelassées les unes dans les autres, que beste ny homme n'y auroit pû passer ${ }^{30}$. Le narrateur n'omet pas de commenter cette mesure magique:

On ne douta point que la Fée n'eust encore fait là un tour de son metier, afin que la Princesse pendant qu'elle dormiroit, n'eust rien à craindre des Curieux ${ }^{31}$.

La facilité avec laquelle le prince promis à la Princesse franchit ce taillis nous amène à penser que cette mesure visait surtout à empêcher qu'un homme ordinaire vienne s'emparer de celle qui était «née pour être couronnée». Il importe en effet de noter que ce «fils de roi » prédestiné à épouser la princesse par "l'Arrêt des Fées" ne devra rien faire de lui même pour l'obtenir. Le chemin du château s'ouvre devant lui sans qu'il ait besoin de traverser les ronces, la princesse se réveille d'elle-même et s'offre à lui sans qu'il ait besoin de la "conquérir", de l'embrasser ou de 
la "sauver» de quelque danger ou de quelque malédiction que ce soit, tout simplement parce que «l'Arrest des Fées l'ordonnoit ainsi ». Le conte de Perrault, loin de l'investir d'un "male-rescuer archetype» comme le prétendent nombre d'interprétations d'inspiration féministe ${ }^{32}$ représente le prince au contraire comme une sorte d'antihéros. Le privilège de sa naissance royale, sur laquelle table l'Arrêt des Fées, le dispense des rudes épreuves des héros des romans chevaleresques qui partent à la conquête périlleuse de princesses tenues prisonnières par d'horribles dragons. Rien de tel pour ce prince privilégié dont la lâcheté et la passivité mettent grandement en péril la vie de la Belle et de leurs enfants.

Une autre mesure magique prise par la Fée confirme le fait que le fameux "Arrest des Fées" censé sauver la vie de la princesse, l'expose au contraire à de nouveaux dangers inconsidérés. Le texte du recueil de 1697 évoque l'accueil plus qu’engageant que la princesse réserve au prince inconnu au moment de son réveil en lui attribuant cette phrase: "[...] est-ce vous, mon Prince, luy dit elle, vous vous estes bien fait attendre ${ }^{33}$. Le narrateur ne manque pas d'ajouter ce commentaire:

Il estoit plus embarassé qu'elle, \& et l'on ne doit pas s'en étonner; elle avoit eu le temps de songer à ce qu'elle auroit à luy dire; car il y a l'apparence, (l'Histoire n'en dit pourtant rien) que la bonne Fée pendant un si long sommeil, luy avoit procuré le plaisir des songes agreables ${ }^{34}$.

Le texte paru un an plus tôt dans le Mercure galant avait toutefois donné une explication plus claire à ce sujet:

Oui, mon cher prince, lui répondit la princesse, je sens bien à votre vue que nous sommes faits l'un pour l'autre. C'est vous que je voyais, que j'entretenais, que j'aimais pendant mon sommeil. La fée m'avait rempli l'imagination de votre image ${ }^{35}$.

Si l'on veut décrire le processus d'un conditionnement psychique, on ne peut pas trouver de formule plus pertinente que celle de la fée ayant "rempli l'imagination" de la princesse de l'image d'un prétendant princier. Elle se serait donc employée à nourrir l'esprit de la princesse pendant

32. Voir à ce sujet la contribution de Donald Haase dans le présent volume.

33. Ch. Perrault, Histoires ou contes du temps passé, p. 19.

34. Ibid., p. 27.

35. Ch. Perrault, Charles Perrault, Contes, éd. R. Zuber, p. 336. 
cent ans de l'idée que l'inconnu au sang royal qui se présenterait un jour devant son lit serait bel et bien l'homme de sa vie et que, par conséquent, elle devait immédiatement se donner à lui sans se préoccuper de savoir qui il était vraiment. S'étant donnée inconditionnellement à ce prince inconnu, la princesse reste en effet dans l'ignorance totale des dangers qui l'attendent, ignorance que les cent ans de retard sur l'état du monde et du royaume ne font qu'aggraver. L'ambiguïté de l'action de la «bonne Fée» apparaît dans le détour d'une phrase: «La bonne Fée qui luy avoit sauvé la vie, en la condamnant à dormir cent ans, [...]» ${ }^{36}$. La suite de l'histoire nous amène à comprendre qu'au moment du départ à la guerre du prince devenu roi, la princesse ignore toujours tout de la nature d'ogresse de sa belle-mère dont les gens ne parlent que "tout bas" ${ }^{37}$. La réponse de la Belle au Maître-d'Hôtel envoyé pour la tuer en dit long sur l'esprit de soumission de celle que les Fées avaient dotée d'un "esprit d'Ange»:

Faites vostre devoir, luy dit-elle, en luy tendant le col; executez l'ordre qu'on vous a donné; j'irai revoir mes enfans, mes pauvres enfans que j'ay tant aimez, car elle les croyoit morts depuis qu'on les avoit enlevez sans luy rien dire.

Comment ne pas percevoir, dans cette optique, l'ironie ${ }^{38}$ du retour tout à fait fortuit de l'époux au moment où sa mère s'apprête à jeter femme et enfants dans la cuve remplie de serpents? C'est donc à un pur hasard que la Belle dotée de "toutes les perfections imaginables" doit sa survie et celle de ses enfants dans un univers dominé par le pouvoir, l'hypocrisie et la cruauté. Au moment de dédier son recueil de contes en prose à Elisabeth Charlotte d'Orléans, s'agit-il pour Perrault de mettre en garde la jeune femme contre ce type de mariage-marchandage pour le moins périlleux? S'agit-il, plus largement, de mettre en garde toutes les filles nubiles des risques encourus par elles dans une société qui subordonne les relations conjugales aux intérêts politiques et financiers que Perrault avait dénoncés dans son Apologie des Femmes de 1694? Cette mise en garde par le biais de récits "enjoués" pourrait bien être la morale vraiment utile et moderne dont l'académicien dote non seulement son premier conte en prose, mais aussi les suivants. Les Histoires ou contes

36. Ch. Perrault, Histoires ou contes du temps passé, p. 13 (je souligne).

37. Ibid., p. 32.

38. Voir à ce sujet l'étude de Jean-Pierre van Elslande, «Parole d'enfant». 
du temps passé. Avec des Moralitez ne découvrent pas leur morale "utile" de façon immédiate, mais à une condition très précise que l'épître des Histoires ou contes $d u$ temps passé indique clairement:

Cependant, MADEMOISELLE, quelque disproportion qu'il y ait entre la simplicité de ces Récits, \& les lumières de votre esprit, si on examine bien ces Contes, on verra que je ne suis pas aussi blamable que je parois d'abord. Ils renferment tous une Morale trés-sensée, \& qui se découvre plus ou moins, selon le degré de pénétration de ceux qui les lisent; [...] ${ }^{39}$.

Ute Heidmann

Université de Lausanne

39. Ch. Perrault, Histoires ou contes du temps passé, p. 3. 


\section{BIBLIOGRAPHIE}

\section{Sources}

Apulée, Les Métamorphoses ou l'Ane d'or, texte établi par D. S. Robertson, émendé, présenté et traduit par Olivier Sers, Paris, Les Belles Lettres, 2007 (coll. classiques en poche).

Perrault, Charles, "Préface», in Griselidis, nouvelle avec le conte de Peau d'Asne, et celuy des Souhaits ridicules, quatrième édition, Paris, Coignard, 1695. Fac-similé Firmin-Didot, Paris, 1929.

-, Contes de Perrault, éd. Gilbert Rouger, Paris, Classiques Garnier, 1967.

—, L'Apologie des Femmes. Par Monsieur P**, fac-similé de l'édition Coignard, Genève, Slatkine Reprints, 1979 [1694].

-, Histoires ou contes du temps passé. Avec des Moralitéz, fac-similé du second tirage de l'édition Barbin, Paris, Firmin Didot, 1929; également Slatkine Reprints, Genève, 1980 [1697].

-, Charles Perrault. Contes, éd. Jean-Pierre Collinet, Paris, Gallimard, coll. Folio, 1981.

-, Charles Perrault. Contes, éd. Roger Zuber, Paris, Imprimerie Nationale, 1987 (coll. Lettres françaises).

-, Contes de Charles Perrault, éd. Marc Escola, Paris, Gallimard, 2005 (Foliothèque 131).

\section{Travaux}

Delarue, Paul, Tenèze, Marie-Louise, Le Conte populaire français, Paris, Maisonneuve \& Larose, 2002.

Elslande, Jean-Pierre van, "Parole d'enfant: Perrault et le déclin du Grand Siècle», PFSCL XXVI, 51 (1999), p. 439-454.

Fenling, Detlev, Amor und Psyche; die Schöpfung des Apuleius und ihre Einwirkung auf das Märchen: eine Kritik der romantischen 
Märchentheorie, Mainz, Akademie der Wissenschaften und der Literatur, 1977.

Gély, Véronique, L'invention d'un mythe: Psyché, Paris, Champion, 2006.

Graham, Anderson, Fairytale in the Ancient World, London/New York, Routledge, 2000.

Heidmann, Ute, "La Barbe bleue palimpseste. Comment Perrault recourt à Virgile, Scarron et Apulée, en réponse à Boileau", Poétique, 154 (2008), p. 161-182.

-, "Comment faire un conte moderne avec un conte ancien? Perrault en dialogue avec Apulée et La Fontaine», Littérature, 153 (2009), p. 19-35.

—, "Enjeux d'une comparaison différentielle et discursive», in Les nouvelles voies du comparatisme, éd. par $\mathrm{H}$. Roland, S. Vanasten, Gent, Academia Press, 2010, p. 27-40 (CLW 2, Cahier voor Literatuurwetenschap).

—, "Quel apport du comparatisme pour l'étude des cultures? L'exemple du Petit Chaperon rouge", in Etudes culturelles, anthropologie culturelle et comparatisme, éd. par A. D. Leiva, S. Hubier, Ph. Chardin, D. Souiller, Dijon, Editions du Murmure, 2010, vol. 1, p. 135-148.

—, «Perrault en dialogue avec Apulée, Fenelon et Lhéritier: Le Petit Chaperon rouge palimpseste", in Il était une fois l'interdisciplinarité. Approches discursives des Contes de Perrault, éd. par Claire Badiou-Monferran, Louvain-la-Neuve, Academia Bruylant, 2010, p. $85-103$.

Heidmann, Ute, Adam, Jean-Michel, Textualité et intertextualité des contes, Paris, Classiques Garnier, 2010.

Huet, Pierre-Daniel, Lettre-traité de Pierre-Daniel Huet sur l'origine des romans, édition du Tricentenaire (1669-1969) suivie de La lecture des vieux romans par Jean Chapelain, édition critique par Fabienne Gégou, Paris, Editions A.-G. Nizet, 1971 [1669].

Robert, Raymonde, Le conte de fées littéraire en France de la fin du XVII à la fin du XVIII e siècle, Paris, Champion, 2002 [1982].

Soriano, Marc, Les contes de Perrault. Culture savante et traditions populaires, Paris, Gallimard, 1968.

Swahn, Jan-Öjwind, The Tale of Cupid and Psyche (Aarne-Thompson 425-428), Lund, Gleerup, 1955. 